\title{
Sulfonylureas as second line drugs in type 2 diabetes and the risk of cardiovascular and hypoglycaemic events: population based cohort study
}

\author{
Antonios Douros, ${ }^{1,2,3}$ Sophie Dell'Aniello, ${ }^{1}$ Oriana Hoi Yun Yu, ${ }^{1,4}$ Kristian B Filion, ${ }^{1,2,5}$ \\ Laurent Azoulay, ${ }^{1,2,6}$ Samy Suissa ${ }^{1,2,5}$
}

${ }^{1}$ Centre for Clinical

Epidemiology, Lady Davis

Institute, Jewish General

Hospital, 3755 Cote Ste-

Catherine, H-461 Montréal, QC

H3T 1E2, Canada

${ }^{2}$ Department of Epidemiology,

Biostatistics, and Occupational

Health, McGill University,

Montréal, QC, Canada

${ }^{3}$ Institute of Clinical

Pharmacology and Toxicology,

Charité-Universitätsmedizin

Berlin, corporate member

of Freie Universität Berlin,

Humboldt-Universität zu Berlin,

and Berlin Institute of Health,

Berlin, Germany

${ }^{4}$ Division of Endocrinology, Jewish General Hospital,

Montréal, QC, Canada

${ }^{5}$ Division of Clinical

Epidemiology, Department of

Medicine, McGill University,

Montréal, QC, Canada

${ }^{6}$ Gerald Bronfman Department of Oncology, McGill University, Montréal, QC, Canada

Correspondence to: S Suissa samy.suissa@mcgill.ca

Additional material is published online only. To view please visit the journal online.

Cite this as: $B M J$ 2018;362:k2693 http://dx.doi.org/10.1136/bmj.k2693

Accepted: 11 June 2018

\section{ABSTRACT}

OBJECTIVE

To assess whether adding or switching to sulfonylureas is associated with an increased risk of myocardial infarction, ischaemic stroke, cardiovascular death, all cause mortality, and severe hypoglycaemia, compared with remaining on metformin monotherapy in patients with type 2 diabetes.

DESIGN

Population based cohort study.

SETTING

General practices contributing data to the UK Clinical Practice Research Datalink.

\section{PARTICIPANTS}

Patients with type 2 diabetes initiating metformin monotherapy between 1998 and 2013.

\section{MAIN OUTCOME MEASURES}

Using the prevalent new-user cohort design we matched 1:1 patients adding or switching to sulfonylureas with those remaining on metformin monotherapy on high-dimensional propensity score, haemoglobin $\mathrm{A} 1 \mathrm{c}$, and number of previous metformin prescriptions. The two groups were compared using Cox proportional hazards models to estimate adjusted hazard ratios and $95 \%$ confidence intervals for the study outcomes.

RESULTS

Among 77138 metformin initiators, 25699 added or switched to sulfonylureas during the study period. During a mean follow-up of 1.1 years, sulfonylureas were associated with an increased risk of myocardial infarction (incidence rate $7.8 v 6.2$ per 1000 person years, hazard ratio $1.26,95 \%$ confidence interval 1.01 to 1.56$)$, all cause mortality $(27.3 \vee 21.5,1.28,1.15$ to 1.44$)$, and severe hypoglycaemia $(5.5 v 0.7,7.60$, 4.64 to 12.44$)$ compared with continuing metformin

\section{WHAT IS ALREADY KNOWN ON THIS TOPIC}

Sulfonylureas are widely used second line oral antidiabetic drugs

Previous studies have assessed their cardiovascular and hypoglycaemic safety as first line drugs or in comparison with other second line antidiabetic drugs

\section{WHAT THIS STUDY ADDS}

Sulfonylureas as second line drugs are associated with an increased risk of myocardial infarction, all cause mortality, and severe hypoglycaemia, compared with remaining on metformin monotherapy

Continuation of metformin when introducing sulfonylureas is safer than switching monotherapy. There was a trend towards increased risks of ischaemic stroke $(6.7 \vee 5.5,1.24,0.99$ to 1.56) and cardiovascular death $(9.4 v 8.1,1.18$, 0.98 to 1.43 ). Compared with adding sulfonylureas, switching to sulfonylureas was associated with an increased risk of myocardial infarction (hazard ratio $1.51,95 \%$ confidence interval, 1.03 to 2.24 ) and allcause mortality $(1.23,1.00$ to 1.50$)$. No differences were observed for ischaemic stroke, cardiovascular death, or severe hypoglycaemia.

\section{CONCLUSIONS}

Sulfonylureas as second line drugs are associated with an increased risk of myocardial infarction, all cause mortality, and severe hypoglycaemia, compared with remaining on metformin monotherapy. Continuing metformin when introducing sulfonylureas appears to be safer than switching.

\section{Introduction}

Sulfonylureas are oral antidiabetic drugs recommended as second line treatment in patients with type 2 diabetes. ${ }^{1}$ Despite the recent approval of several new drugs, sulfonylureas remain the most commonly prescribed antidiabetic drugs after treatment failure with the first line drug metformin. ${ }^{2}$ The safety of sulfonylureas with respect to adverse cardiovascular and hypoglycaemic events has been studied extensively. ${ }^{34}$ However, studies focusing specifically on their cardiovascular and hypoglycaemic safety as a second line drug in patients with poorly controlled diabetes in need of adding or switching to other drugs, are sparse and limited.

The randomised controlled trials evaluating sulfonylureas as second line drugs were underpowered to assess cardiovascular complications of diabetes or severe hypoglycaemia, which may contribute to the development of adverse cardiovascular events. ${ }^{56}$ In clinical practice, most observational studies assessing these outcomes compared sulfonylureas with other second line antidiabetic drugs such as dipeptidyl peptidase-4 inhibitors or insulin. ${ }^{7-9}$ Thus, the specific risk of supplementing treatment with sulfonylureas compared with staying on metformin monotherapy has rarely been investigated, and several of the respective observational studies had method limitations such as selection bias, ${ }^{10}{ }^{11}$ exposure misclassification, ${ }^{12}$ and residual confounding. ${ }^{10} 12$

Metformin is associated with a decreased risk of cardiovascular events and low rates of hypoglycaemia. ${ }^{13}$ Supplementing treatment with sulfonylureas, a potentially cardiotoxic class with 
high risk of hypoglycaemic events, ${ }^{3}{ }^{14}$ may outweigh the benefits of metformin. Thus, the objective of our population based study was to assess whether the use of second line sulfonylureas, after metformin, is associated with increased risks of myocardial infarction, ischaemic stroke, cardiovascular death, all cause mortality, and severe hypoglycaemia in patients with type 2 diabetes, compared with continuation of metformin monotherapy.

\section{Methods}

\section{Data sources}

We used the UK Clinical Practice Research Datalink (CPRD) linked to the Hospital Episode Statistics (HES) and Office for National Statistics (ONS) databases. The CPRD is a large primary care database which contains the medical records for over 14 million people registered at over 680 general practices. ${ }^{15}$ Diagnoses and procedures are recorded using the Read code classification. Drugs prescribed by general practitioners are coded based on the UK Prescription Pricing Authority dictionary. The CPRD contains information on anthropometric variables such as body mass index, and lifestyle variables such as smoking and alcohol use. CPRD data have been previously validated and shown to be of high quality. ${ }^{16}$ The HES contains all inpatient and outpatient hospital admission information, including primary and secondary diagnoses (coded using ICD-10 (international classification of diseases, 10th revision), and hospital procedures (coded using the Office of Population Censuses and Surveys classification of interventions and procedures, 4th version (OPCS-4)). The ONS database contains the electronic death certificates of all UK residents and includes the underlying cause of death (coded using ICD-9 and ICD-10). HES and ONS data can be linked to the CPRD since 1 April 1997, and are limited to general practices in England that have consented to the linkage scheme (currently representing $75 \%$ of all practices in England). ${ }^{16}$

\section{Base cohort}

The methods have been previously reported. ${ }^{17}$ We first formed a base cohort of patients newly treated with metformin in monotherapy for type 2 diabetes between 1 April 1998 and 31 March 2013, with follow-up until 31 March 2014. Base cohort entry was defined by the date of the first metformin prescription. We excluded all patients aged under 40, those with less than one year of medical history in the CPRD before cohort entry, and women with a diagnosis of polycystic ovary syndrome at any time before cohort entry. We also excluded patients prescribed any antidiabetic drugs at any time before base cohort entry. Patients with a history of cardiovascular disease at cohort entry were included.

\section{Study cohort}

The study cohort was formed by identifying all subjects from the base cohort of metformin initiators who subsequently added or switched to a sulfonylurea as second line treatment. Patients who added or switched to other antidiabetic drugs were censored. For each patient adding or switching to a sulfonylurea, we identified a matched reference patient who also was a metformin initiator but remained on metformin, using a prevalent newuser design. ${ }^{18}$ The potential reference patients were selected from the corresponding exposure sets, namely from the metformin initiators in the base cohort who received a metformin prescription within three months of the date the exposed patients added or switched to a sulfonylurea, and who received the same number of metformin prescriptions during the time in the base cohort as the exposed subjects (see efigure 1, supplementary materials). Thus, the number of metformin prescriptions between base cohort entry (monotherapy initiation) and study cohort entry (adding or switching to a sulfonylurea or matched continuation) was inherently a matching covariate. Moreover, exposed and reference subjects were matched on haemoglobin A1c level ( $\leq 7 \%, 7.1$ $8 \%,>8 \%$, or unknown) at study cohort entry. Finally, exposed and reference subjects were matched on high-dimensional propensity score. ${ }^{19}$ The highdimensional propensity score method empirically selects covariates based on their prevalence and potential for confounding. For each member of each matched set, we identified all available information from seven data dimensions (five dimensions from the CPRD: drug prescriptions, procedures, diagnoses, disease history, and administrative information; two dimensions form the HES: diagnoses and procedures) in the one year period before the date of the matched set. We then applied conditional logistic regression to estimate the propensity of receiving a sulfonylurea drug, thereby considering the 500 most likely confounders. Patients with non-overlapping high-dimensional propensity scores were trimmed from the cohort. The high-dimensional propensity score procedure was repeated for each outcome, since this method calculates a bias term that accounts for the association with a specific outcome. Matched metformin patients could add or switch to sulfonylureas later during follow-up. In this case, the follow-up for the metformin monotherapy group was censored at the point of adding or switching to sulfonylureas. Then, the patient was included as a sulfonylurea user from that point onwards and could be potentially matched to a metformin patient.

Patients meeting the study inclusion criteria were followed until the earliest of the following events: treatment discontinuation, occurrence of one of the study outcomes, end of registration with the general practice, or end of the study period (31 March 2014). Treatment discontinuation was defined either by the absence of a new prescription by the end of a 60 day period (30 days prescription duration plus 30 days grace period) or by the addition or the switch to another, non-sulfonylurea antidiabetic drug. For the metformin initiators adding or switching to sulfonylureas, further switches within the sulfonylurea class were permitted. 


\section{Study outcomes}

We considered five outcomes: hospital admission for myocardial infarction, hospital admission for ischaemic stroke, cardiovascular death, all cause mortality, and severe hypoglycaemia. Hospital admission for myocardial infarction (ICD-9 codes 410.x, ICD-10 codes I21.x) and ischaemic stroke (ICD9 codes 433.x, 434.x, or 436.x; ICD-10 codes I63.x or I64.x) were identified using the HES and ONS. The diagnostic codes to identify myocardial infarction in HES have been shown to be highly valid (>90\%). ${ }^{20}$ The validity of stroke diagnoses in administrative data are also high $(>80 \%) .{ }^{21}$ Cardiovascular death (ICD-9 codes 390.x-398.x, 401.x-405.x, 410.x-417.x, 420.x-429.x (except 427.5), 430.x-438.x, or 440.x-447.x; ICD-10 codes I00.x-I77.x (except I46.9)) was identified in ONS, and all cause mortality was identified from all three databases, with the date of death defined by the earliest recording of death in any database. Severe hypoglycaemia (ICD-10 codes E16.0, E16.1, E16.2) was identified in HES.

\section{Statistical analysis}

We used descriptive statistics to summarise the characteristics of the patients in the matched groups. Potential imbalances after matching among covariates were assessed using standardised mean differences. Incidence of each outcome was calculated based on the Poisson distribution and expressed as number of events per 1000 person years. Moreover, we constructed a Cox proportional hazards regression model for each outcome that estimated the hazard ratio and the $95 \%$ confidence intervals for sulfonylurea versus metformin. To maximise comparability between the two groups, the models for myocardial infarction, ischaemic stroke, cardiovascular death, and severe hypoglycaemia were additionally adjusted for age, sex, deciles of high-dimensional propensity score, and history of the respective outcome in the year before cohort entry (or, for the case of cardiovascular death, history of myocardial infarction or ischaemic stroke). The model for all cause mortality was additionally adjusted for age, sex, and deciles of high-dimensional propensity score.

\section{Secondary analyses}

We conducted three secondary analyses. Firstly, we assessed the risk of the study outcomes separately for addition of sulfonylureas to metformin and for switching to sulfonylureas from metformin. For this analysis, we used a time dependent exposure definition subcategorising the person time of sulfonylurea use, which resulted in three mutually exclusive categories: current use of metformin only (reference), current use of sulfonylureas only, and concomitant current use of metformin and sulfonylureas. Thus, the same patient could contribute person time to different exposure categories. Secondly, to assess a duration-response relation between adding or switching to sulfonylureas and the risk of each study outcome, drug use was further categorised according to three predefined durations ( $\leq 3$ months, 3.1-12 months, $>12$ months). Thirdly, given the pharmacologic heterogeneity observed among different sulfonylureas, the risk of each study outcome was assessed separately for two groups of sulfonylureas classified by duration of action and pancreas specificity. The first group included pancreas non-specific, long acting drugs (ie, glyburide and glimepiride). The second group included pancreas specific, short acting drugs (ie, gliclazide, glipizide, and tolbutamide). ${ }^{17} 22-25$ This analysis was based on the first sulfonylurea added or switched to. Switches among sulfonylureas of the same group were allowed during follow-up.

\section{Sensitivity analyses}

We performed four sensitivity analyses to assess the robustness of our findings. Firstly, to assess possible exposure misclassification, we repeated the analyses using a 60 day grace period between nonoverlapping successive prescriptions. Secondly, the analyses for myocardial infarction, ischaemic stroke, cardiovascular death, and severe hypoglycaemia were repeated after excluding patients with a history of the outcome (or, for the case of cardiovascular death, history of myocardial infarction or ischaemic stroke) in the year before cohort entry. Thirdly, to assess the potential impact of residual confounding, we repeated the primary analysis after additionally adjusting for covariates with a standardised mean difference $>5 \%$. Finally, to assess the potential impact of unmeasured confounding, we conducted a post-hoc sensitivity analysis using the approach proposed by Ding and VanderWeele (described in eMethods 1 , supplementary material). ${ }^{26}$

\section{Negative control analysis}

To further assess the validity of our findings, we conducted an additional analysis using a negative control outcome. ${ }^{27}$ We compared metformin initiators who added or switched to sulfonylureas with metformin initiators who stayed on metformin monotherapy regarding the risk of diabetic retinopathy (identified using Read codes from the CPRD and ICD-10 codes from the HES), since no differential effects between metformin and sulfonylureas have been reported for this outcome. ${ }^{28}$ For this analysis, we additionally excluded all patients with previous retinopathy in order to assess incident disease.

\section{Ancillary analysis}

In an ancillary analysis, we compared head-to-head patients adding sulfonylureas to metformin with patients switching to sulfonylureas from metformin. For this analysis, we defined addition of sulfonylureas as a metformin prescription in the first month after the first sulfonylurea prescription. Switching to sulfonylureas was defined by the absence of a metformin prescription in the same period. Next, we performed a multivariable logistic regression to estimate the probability (propensity score) of switching to sulfonylureas versus adding sulfonylureas conditional on all covariates 
listed in the manuscript. We then trimmed patients with non-overlapping propensity score distributions. The remaining patients were followed from one month after the initial sulfonylurea prescription until they added or switched to a non-metformin, non-sulfonylurea antidiabetic drug or experienced one of the study outcomes, whichever occurred earlier. Patients adding sulfonylureas were additionally censored at metformin or sulfonylurea discontinuation, and patients switching to sulfonylureas were additionally censored in case of metformin re-initiation or sulfonylurea discontinuation. The hazard ratio of the study outcomes was estimated using a Cox proportional hazards model adjusted for age, sex, history of the outcome in the year before cohort entry (or, for the case of cardiovascular death, history of myocardial infarction or ischaemic stroke), propensity score deciles, and for covariates with a standardised mean difference $>5 \%$. All analyses were conducted with SAS version 9.4 (SAS Institute, Cary, NC) and R (R Foundation for Statistical Computing, Vienna, Austria).

\section{Patient involvement}

No patients were involved in setting the research question or the outcome measures, nor were they involved in developing plans for design or implementation of the study. No patients were asked to advise on interpretation or writing up of results. There are no plans to disseminate the results of the research to study participants or the relevant patient community.

\section{Results}

Figure 1 shows that the base cohort included 77138 patients with a first prescription for metformin between 1 April 1998 and 31 March 2013. A total of 25699 patients added or switched to sulfonylureas during follow-up. For the analysis on all cause mortality, 2107 (8\%) of these patients were trimmed from the cohort due to non-overlapping high-dimensional propensity score distributions or absence of eligible matches. Figure 1 shows that the study cohort for this outcome was 23592 patients who added or switched to sulfonylureas and 23592 matched patients who remained on metformin monotherapy. The size of the study cohorts for the other four outcomes was similar.

The mean follow-up was 1.1 (SD 1.4) years, generating a total of 244150 patient years. During follow-up, there were 337 myocardial infarctions (incidence rate 6.9 per 1000 patient years, 95\% confidence interval 6.2 to 7.7$), 299$ ischaemic strokes $(6.1,5.5$ to 6.9$), 429$ cardiovascular deaths $(8.7,7.9$ to 9.6$), 1190$ deaths from any cause (24.4, 23.0 to 25.8 ), and 150 severe hypoglycaemic events (3.1, 2.6 to 3.6). The most frequent causes of death were cancer $(31 \%)$, cardiovascular diseases (31\%), and respiratory diseases (10\%). Table 1 shows the baseline characteristics of the matched cohorts for the analysis on all cause mortality. Corresponding tables for the other outcomes (myocardial infarction, ischaemic stroke, cardiovascular death, and severe hypoglycaemia) were practically identical (see eTables 1-4, supplementary materials). After high-dimensional propensity score matching, patients adding or switching to sulfonylureas had a similar baseline profile to those remaining on metformin monotherapy.

Table 2 and eFigures 2-6 (supplementary materials) show the results for the five outcomes. Compared with the use of metformin monotherapy, adding or switching to sulfonylureas was associated with an increased risk of myocardial infarction (7.8 $v 6.2$ per 1000 person years, hazard ratio $1.26,95 \%$ confidence interval 1.01 to 1.56$)$, all cause mortality ( $27.3 v 21.5$, $1.28,1.15$ to 1.44 ), and severe hypoglycaemia (5.5 $v 0.7,7.60,4.64$ to 12.44$)$. There was also a trend towards increased risks of ischaemic stroke (6.7 $v 5.5$, $1.24,0.99$ to 1.56$)$ and cardiovascular death (9.4 $\mathrm{v}$ $8.1,1.18,0.98$ to 1.43$)$.

Separately comparing adding and switching to sulfonylureas suggested that the increase in the risk was driven by the switching and not the addition for myocardial infarction, cardiovascular death, and all cause mortality, but not for ischaemic stroke or severe hypoglycaemia (see eTable 5, supplementary materials). The analyses based on different durations of use yielded higher point estimates for all five outcomes for shorter durations of use and especially for the $\leq 3$ months category (see eTables 6-10, supplementary materials). Classifying sulfonylureas based on important pharmacologic properties provided similar point estimates for the two sulfonylurea groups (see eTable 11, supplementary materials).

Figure 2 shows that the results of the primary analysis remained consistent in the sensitivity analyses (see full detail in eTables 12-14, supplementary materials). For myocardial infarction, the extension of the grace period to 60 days led to a dilution of the hazard ratio, resulting in a non-statistically significant association (hazard ratio 1.12, 95\% confidence interval 0.94 to 1.32). Based on a post-hoc analysis, the findings of the primary analysis on myocardial infarction, all cause mortality, and severe hypoglycaemia are unlikely to be the result of an unmeasured confounder under most plausible exposure-confounder and confounder-outcome associations (see eTables 1517, supplementary materials). Finally, we observed no difference in the risk among patients taking sulfonylureas and metformin regarding the negative control outcome of diabetic retinopathy (incidence rate $41.4 v 40.4$ per 1000 person years, hazard ratio 1.02, $95 \%$ confidence interval 0.92 to 1.14 ).

Table 3 shows the results of the head-to-head comparison between patients adding sulfonylureas and patients switching to sulfonylureas (baseline characteristics for the five outcomes are presented in eTables 18-22, supplementary materials). Compared with adding sulfonylureas, switching to sulfonylureas was associated with an increased risk of myocardial infarction (hazard ratio 1.51, 95\% confidence interval 1.03 to 2.24) and a borderline increased risk of all cause mortality $(1.23,1.00$ to 1.50$)$. No differences in risk were observed for ischaemic stroke $(0.88,0.58$ 
Patients with a first prescription of metformin between 1 April 1998 and 31 March 2013 and linkable to HES and ONS

\section{i 137043}

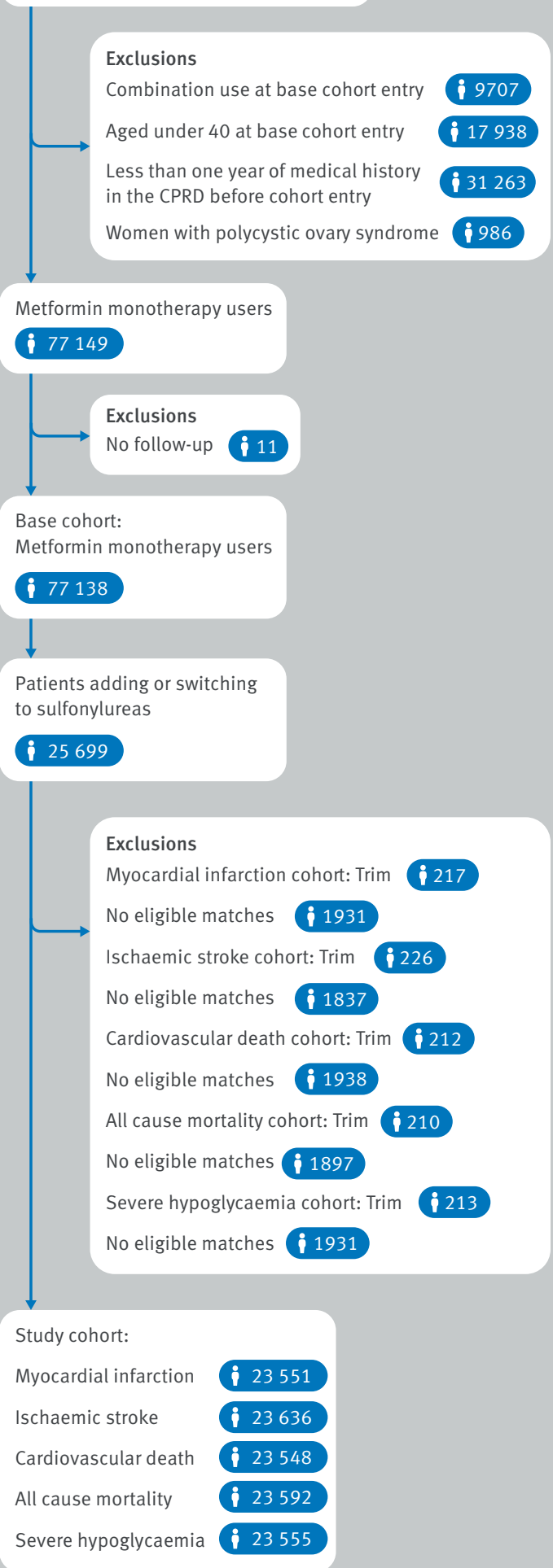

Fig 1 | Flowchart showing the base and study cohorts. HES=Hospital Episode Statistics; ONS=Office for National Statistics; CPRD=Clinical Practice Research Datalink. to 1.33$)$, cardiovascular death $(1.22,0.87$ to 1.71$)$, or severe hypoglycaemia (1.06, 0.65 to 1.71$)$.

\section{Discussion}

Our study assessed the cardiovascular and hypoglycaemic risk associated with the use of sulfonylureas as second line drugs. Among metformin initiators, adding or switching to sulfonylureas was associated with an increased risk of myocardial infarction, all cause mortality, and severe hypoglycaemia, compared with patients remaining on metformin. Moreover, there was a trend towards increased risks of ischaemic stroke and cardiovascular death. Importantly, the observed associations with myocardial infarction and all cause mortality were driven by the switching to sulfonylureas and not the addition of sulfonylureas. The results of the primary analysis remained consistent in sensitivity analyses, as well as after classifying sulfonylureas in two different groups based on important pharmacologic properties.

\section{Comparison with other studies}

Numerous observational studies have evaluated the safety of sulfonylureas as first line drugs. ${ }^{3}$ However, their results cannot be directly extrapolated to patients having failed initial monotherapy with metformin, since these patients probably have progressive disease and are thus at a higher risk of adverse cardiovascular and hypoglycaemic events. Most of the studies evaluating the safety of sulfonylureas as second line drugs used other second to third line drugs as comparators. $^{7-9}$ Thus, no conclusions can be drawn on the risk of adding or switching to sulfonylureas, which have been associated with an increased risk of cardiovascular and hypoglycaemic events, ${ }^{3}{ }^{14}$ as compared with remaining on monotherapy with metformin, a drug with potential cardioprotective properties and low risk of hypoglycaemia. ${ }^{13}$ Moreover, among the few studies assessing the latter risk, several featured method limitations such as selection bias owing to the inclusion of prevalent users, ${ }^{10}{ }^{11}$ exposure misclassification, ${ }^{12}$ and confounding. ${ }^{10}{ }^{12}$ For our study, we used the recently developed prevalent newuser design. ${ }^{18}$ To emulate the randomised controlled trial, this design identifies (at the doctor visit that led to the patient on metformin adding or being switched to sulfonylureas) a comparable patient with the same history of metformin use and of other characteristics, but who on that visit continued on metformin. Thus, we could specifically address the question of how supplementing treatment with sulfonylureas performs in patients for who metformin monotherapy has failed.

This is the first observational study assessing the risk of myocardial infarction associated with sulfonylureas as second line treatment. Several potential mechanisms could account for the increase in the risk compared with patients remaining on metformin monotherapy. Firstly, sulfonylureas are associated with weight gain, which is an important risk factor for myocardial infarction. ${ }^{25}$ Secondly, hypoglycaemia is implicated 


\begin{tabular}{|c|c|c|c|}
\hline Characteristic & Sulfonylureas $(n=23592)$ & Metformin ( $n=23592)$ & Standardised mean difference (\%) \\
\hline Mean (SD) age (years) & $64(12)$ & $64(12)$ & 3.6 \\
\hline Women & $10269(43.5)$ & $10018(42.5)$ & 2.2 \\
\hline Mean (SD) diabetes duration (years) & $3.5(3.6)$ & $3.4(3.5)$ & 2.8 \\
\hline Alcohol related disorders & $1284(5.4)$ & $1274(5.4)$ & 0.2 \\
\hline \multicolumn{4}{|l|}{ Smoking status: } \\
\hline Ever & $12944(54.9)$ & $12810(54.3)$ & 1.1 \\
\hline Never & $10435(44.2)$ & $10559(44.8)$ & -1.1 \\
\hline Unknown & $213(0.9)$ & $223(0.9)$ & -0.4 \\
\hline \multicolumn{4}{|l|}{ Body mass index $\left(\mathrm{kg} / \mathrm{m}^{2}\right)$ : } \\
\hline$<25$ & $2694(11.4)$ & $2182(9.2)$ & 7.1 \\
\hline $25-30$ & $7914(33.5)$ & 7559 (32.0) & 3.2 \\
\hline$\geq 30$ & $12554(53.2)$ & $13425(56.9)$ & -7.4 \\
\hline Unknown & $430(1.8)$ & $426(1.8)$ & 0.1 \\
\hline Mean (SD) haemoglobin A1c (\%) & $8.7(1.7)$ & $8.4(1.5)$ & \\
\hline \multicolumn{4}{|l|}{ Haemoglobin A1c (\%): } \\
\hline$\leq 7$ & $2028(8.6)$ & $2028(8.6)$ & $t$ \\
\hline $7.1-8.0$ & $7106(30.1)$ & $7106(30.1)$ & + \\
\hline$>8$ & $12439(52.7)$ & $12439(52.7)$ & + \\
\hline Unknown & $2019(8.6)$ & $2019(8.6)$ & + \\
\hline \multicolumn{4}{|l|}{ Medical history: } \\
\hline Heart failure & $1623(6.9)$ & $1413(6.0)$ & 3.6 \\
\hline Arterial hypertension & $15089(64.0)$ & $14804(62.8)$ & 2.5 \\
\hline Coronary artery disease & $5174(21.9)$ & $4994(21.2)$ & 1.9 \\
\hline Atrial fibrillation or flutter & $2217(9.4)$ & $2052(8.7)$ & 2.4 \\
\hline Hyperlipidaemia & $7424(31.5)$ & $7212(30.6)$ & 1.9 \\
\hline Chronic obstructive pulmonary disease & $3396(14.4)$ & 3275 (13.9) & 1.5 \\
\hline Cancer & $2328(9.9)$ & $2211(9.4)$ & 1.7 \\
\hline Anaemia & $399(1.7)$ & $360(1.5)$ & 1.3 \\
\hline Thyroid disease & $2526(10.7)$ & $2396(10.2)$ & 1.8 \\
\hline Severe hypoglycaemia & $35(0.1)$ & $18(0.0)$ & 2.2 \\
\hline Myocardial infarction & $1190(5.0)$ & $1020(4.3)$ & 3.4 \\
\hline Ischaemic stroke & $434(1.8)$ & $385(1.6)$ & 1.6 \\
\hline \multicolumn{4}{|l|}{ Drugs: } \\
\hline Angiotensin converting enzyme inhibitors & $10523(44.6)$ & $10037(42.5)$ & 4.2 \\
\hline Angiotensin II receptor blockers & $3300(14.0)$ & $3108(13.2)$ & 2.4 \\
\hline Beta-blockers & $6163(26.1)$ & $5751(24.4)$ & 4.0 \\
\hline Calcium channel blockers & $6751(28.6)$ & $6366(27.0)$ & 3.6 \\
\hline Diuretics & $8410(35.6)$ & $7924(33.6)$ & 4.3 \\
\hline Cardiac glycosides & $1069(4.5)$ & $962(4.1)$ & 2.2 \\
\hline Nitrates & $1453(6.2)$ & $1348(5.7)$ & 1.9 \\
\hline Statins & $16402(69.5)$ & $15800(67.0)$ & 5.5 \\
\hline Acetylsalicylic acid & 9187 (38.9) & $8886(37.7)$ & 2.6 \\
\hline Clopidogrel & $875(3.7)$ & $693(2.9)$ & 4.3 \\
\hline Warfarin & $1368(5.8)$ & $1228(5.2)$ & 2.6 \\
\hline Paracetamol & $8350(35.4)$ & 7845 (33.3) & 4.5 \\
\hline Non-steroidal anti-inflammatory drugs & $4987(21.1)$ & $4981(21.1)$ & 0.1 \\
\hline Opioids & $7544(32.0)$ & $7019(29.8)$ & 4.8 \\
\hline \multicolumn{4}{|l|}{ Diabetic complications: } \\
\hline Neuropathy & $3040(12.9)$ & $2888(12.2)$ & 1.9 \\
\hline Peripheral vascular disease & $1439(6.1)$ & $1297(5.5)$ & 2.6 \\
\hline Nephropathy & $3082(13.1)$ & 2561(10.9) & 6.8 \\
\hline Retinopathy & $5566(23.6)$ & $5433(23.0)$ & 1.3 \\
\hline
\end{tabular}

*The two groups were matched 1:1 on high-dimensional propensity score, level of haemoglobin A1c, and number of metformin prescriptions before the first sulfonylurea prescription. Slight differences exist in the populations for each outcome due to trimming.

tMatching variable.

in the development of arrhythmias and cardiac ischaemia, $^{29}$ so the hypoglycaemic propensity of sulfonylureas could contribute to the increased risk of myocardial infarction. The higher estimates observed for shorter durations of use argue for an involvement of short term mechanisms such as arrhythmias and 


\begin{tabular}{|c|c|c|c|c|c|c|}
\hline Exposure & No of patients & No of events & Person years & $\begin{array}{l}\text { Incidence rate }(95 \% \mathrm{Cl}) \text { per } \\
1000 \text { person years }\end{array}$ & Crude hazard ratio $(95 \% \mathrm{Cl})$ & Adjusted hazard ratio $(95 \% \mathrm{Cl})^{*}$ \\
\hline \multicolumn{7}{|c|}{ Myocardial infarction } \\
\hline Metformin & 23551 & 152 & 24673 & $6.2(5.3$ to 7.2$)$ & Reference & Reference \\
\hline Sulfonylureas & 23551 & 185 & 23858 & 7.8 (6.7 to 9.0$)$ & $1.25(1.01$ to 1.55$)$ & $1.26(1.01$ to 1.56$)$ \\
\hline \multicolumn{7}{|c|}{ Ischaemic stroke } \\
\hline Metformin & 23636 & 137 & 24791 & $5.5(4.7$ to 6.5$)$ & Reference & Reference \\
\hline Sulfonylureas & 23636 & 162 & 24015 & 6.7 (5.8 to 7.9$)$ & $1.22(0.97$ to 1.53$)$ & 1.24 (0.99 to 1.56$)$ \\
\hline \multicolumn{7}{|c|}{ Cardiovascular death } \\
\hline Metformin & 23548 & 203 & 25176 & 8.1 (7.0 to 9.3$)$ & Reference & Reference \\
\hline Sulfonylureas & 23548 & 226 & 24011 & $9.4(8.3$ to 10.7$)$ & $1.17(0.97$ to 1.41$)$ & 1.18 (0.98 to 1.43$)$ \\
\hline \multicolumn{7}{|c|}{ All cause mortality } \\
\hline Metformin & 23592 & 533 & 24742 & 21.5 (19.8 to 23.5) & Reference & Reference \\
\hline Sulfonylureas & 23592 & 657 & 24060 & $27.3(25.3$ to 29.5$)$ & 1.27 (1.13 to 1.42$)$ & $1.28(1.15$ to 1.44$)$ \\
\hline \multicolumn{7}{|c|}{ Severe hypoglycaemia } \\
\hline Metformin & 23555 & 18 & 24905 & 0.7 (0.5 to 1.1$)$ & Reference & Reference \\
\hline Sulfonylureas & 23555 & 132 & 23919 & $5.5(4.7$ to 6.5$)$ & $7.59(4.64$ to 12.43$)$ & $7.60(4.64$ to 12.44$)$ \\
\hline
\end{tabular}

against long term mechanisms such as weight gain. Moreover, the similar estimates obtained for the two sulfonylurea groups classified by pancreas specificity indicate that this pharmacodynamic property does not necessarily translate into improved clinical outcomes. ${ }^{17}$ Finally, the absence of an increased risk of myocardial infarction associated with the addition of sulfonylureas to metformin (ie, in case of metformin continuation) alludes to the established beneficial effects of the biguanide in this regard. ${ }^{13}$ Interestingly, metformin was recently shown to also positively modify the cardiovascular effects of a newer class of antidiabetic drugs, dipeptidyl peptidase-4 inhibitors. ${ }^{30}$

Our results on all cause mortality support a previous study showing an increased risk associated with using sulfonylureas only, but not with concomitant use of sulfonylureas and metformin, when compared with using metformin only. ${ }^{31}$ Again, the absence of an increased risk associated with the addition of sulfonylureas to metformin could reflect the beneficial effects of the biguanide. ${ }^{13}$ Moreover, our higher point estimates for shorter durations of use indicate that

\begin{tabular}{l}
\hline Analyses \\
\hline Myocardial Infarction \\
Primary \\
60 day grace period \\
Excluding patients with a history of the outcome \\
Adjusting for additional covariates \\
Ischaemic stroke \\
Primary \\
60 day grace period \\
Excluding patients with a history of the outcome
\end{tabular}

Fig 2 | Forest plot summarising the primary analysis and all sensitivity analyses 


\begin{tabular}{|c|c|c|c|c|c|c|}
\hline Exposure & No of patients & No of events & Person years & $\begin{array}{l}\text { Incidence rate }(95 \% \mathrm{Cl}) \text { per } \\
1000 \text { person years }\end{array}$ & Crude hazard ratio $(95 \% \mathrm{Cl})$ & Adjusted hazard ratio $(95 \% \mathrm{Cl})^{\star}$ \\
\hline \multicolumn{7}{|l|}{ Myocardial infarction } \\
\hline Adding sulfonylureas & 13203 & 57 & 11442 & $5.0(3.8$ to 6.5$)$ & Reference & Reference \\
\hline Switching to sulfonylureas & 9759 & 68 & 5138 & 13.2 (10.4 to 16.8$)$ & 2.65 (1.86 to 3.78$)$ & 1.51 (1.03 to 2.24$)$ \\
\hline \multicolumn{7}{|l|}{ Ischaemic stroke } \\
\hline Adding sulfonylureas & 13300 & 63 & 11542 & $5.5(4.3$ to 7.0$)$ & Reference & Reference \\
\hline Switching to sulfonylureas & 9771 & 46 & 5185 & $8.9(6.6$ to 11.8$)$ & $1.60(1.09$ to 2.34$)$ & $0.88(0.58$ to 1.33$)$ \\
\hline \multicolumn{7}{|l|}{ Cardiovascular death } \\
\hline Adding sulfonylureas & 13217 & 75 & 11464 & $6.5(5.2$ to 8.2$)$ & Reference & Reference \\
\hline Switching to sulfonylureas & 9779 & 93 & 5204 & 17.9 (14.6 to 21.9$)$ & $2.70(1.99$ to 3.66$)$ & $1.22(0.87$ to 1.71$)$ \\
\hline \multicolumn{7}{|l|}{ All cause mortality } \\
\hline Adding sulfonylureas & 13242 & 217 & 11504 & 18.9 (16.5 to 21.5$)$ & Reference & Reference \\
\hline Switching to sulfonylureas & 9800 & 256 & 5216 & $49.1(43.4$ to 55.5$)$ & 2.57 (2.14 to 3.08) & $1.23(1.00$ to 1.50$)$ \\
\hline \multicolumn{7}{|l|}{ Severe hypoglycaemia } \\
\hline Adding sulfonylureas & 13215 & 39 & 11440 & $3.4(2.5$ to 4.7$)$ & Reference & Reference \\
\hline Switching to sulfonylureas & 9770 & 45 & 5177 & $8.7(6.5$ to 11.6$)$ & $2.61(1.70$ to 4.01$)$ & $1.06(0.65$ to 1.71$)$ \\
\hline
\end{tabular}

short term mechanisms such as arrhythmias or seizures and falls potentially induced by severe hypoglycaemia could be involved in the increased risk of mortality.

Our results on severe hypoglycaemia are concordant with a recently published observational study showing an increased risk for second line sulfonylureas. ${ }^{11}$ The similar estimates we obtained for the two groups of sulfonylureas classified by the duration of action argue against an effect of this pharmacokinetic property on the risk of severe hypoglycaemia in the setting of second line treatment. This contrasts with our recent findings on the safety of sulfonylureas as first line drugs, ${ }^{17}$ and underscores the importance of diabetes severity as a possible effect modifier on the risk of adverse events.

\section{Strengths and weaknesses of our study}

Our study has several strengths. Firstly, the population based design, the inclusion of patients with previous events, and the few exclusion criteria make its results highly generalisable. Secondly, the large sample size allowed the calculation of precise estimates even for rare outcomes such as severe hypoglycaemia. Thirdly, our separate analysis of pancreas specific, short acting and pancreas non-specific, long acting sulfonylureas could account for the high pharmacologic heterogeneity observed within this drug class. ${ }^{25}$

Our study also has some limitations. Firstly, owing to its observational nature there is the potential for residual confounding. However, we went to great lengths to minimise this potential bias by matching on high-dimensional propensity score, the number of previous metformin prescriptions, and haemoglobin A1c level. Moreover, we observed no difference in the risk regarding our negative control outcome, diabetic retinopathy. Secondly, owing to the relatively short duration of follow-up, we were not able to assess long term risk differences between the two groups. However, the length of follow-up reflects real world use of second line sulfonylureas. ${ }^{7}$ Thirdly, since metformin use is contraindicated in patients with severe kidney disease and decompensated heart failure, ${ }^{32}$ we cannot exclude that such conditions leading to metformin discontinuation and switching to sulfonylureas may also account for the observed increased risks. Finally, drug dose was not considered in our analyses. Thus, the increased risks observed in patients switching to sulfonylureas compared with patients adding sulfonylureas could also result from the potentially higher sulfonylurea doses in the former group.

\section{Conclusions}

Our study showed an increased risk of myocardial infarction, all cause mortality, and severe hypoglycaemia associated with the use of second line sulfonylureas compared with remaining on metformin monotherapy. The associations with myocardial infarction and all cause mortality were driven by the switching to sulfonylureas and not the addition of sulfonylureas. Thus, in line with current recommendations on the treatment of type 2 diabetes, ${ }^{1}$ continuing metformin when introducing sulfonylureas is safer than switching.

Contributors: AD, SD, OHYY, KBF, LA, and SS contributed to the study concept and design, analysed and interpreted the data, and critically revised the manuscript. AD drafted the manuscript. AD, SD, and SS conducted the statistical analysis. SS acquired the data, obtained funding, and supervised the study. SS is the guarantor.

Funding: $A D$ is the recipient of a Research Fellowship from the German Research Foundation (Deutsche Forschungsgemeinschaft). KBF holds a Chercheur Boursier award from the Fonds de recherche du Québec-Santé (FRQS). LA holds a Chercheur Boursier award from the FRQS and is the recipient of a William Dawson Scholar award. SS is the recipient of the James McGill Professorship award.

Competing interests: All authors have completed the ICMJE uniform disclosure form at www.icmje.org/coi_disclosure.pdf and declare: this research was funded in part by grants from the Canadian Institutes of Health Research, the Canadian Foundation for Innovation, and Boehringer Ingelheim. Boehringer Ingelheim were provided with the opportunity to comment on the manuscript, but they were not directly involved in the design and conduct of the study; the collection, 
management, analysis, and interpretation of the data; or the preparation, review, or approval of the manuscript. SS has received research grants and has participated in advisory board meetings or as a speaker at conferences for AstraZeneca, Bayer, Boehringer Ingelheim, Bristol Myers Squibb, Merck, and Novartis. No other potential conflicts of interest relevant to this article were reported. Ethical approval: The study protocol was approved by the Independent Scientific Advisory Committee of the CPRD (protocol 14_019AMn) and by the Research Ethics Board of the Jewish General Hospital, Montréal, Canada.

Data sharing: No additional data are available.

Transparency: The manuscripts guarantor (SS) affirms that the manuscript is an honest, accurate, and transparent account of the study being reported; that no important aspects of the study have been omitted; and that any discrepancies from the study as planned (and, if relevant, registered) have been explained.

This is an Open Access article distributed in accordance with the Creative Commons Attribution Non Commercial (CC BY-NC 4.0) license, which permits others to distribute, remix, adapt, build upon this work non-commercially, and license their derivative works on different terms, provided the original work is properly cited and the use is noncommercial. See: http://creativecommons.org/licenses/by-nc/4.0/.

1 American Diabetes Association. 7. Approaches to Glycemic Treatment. Diabetes Care 2016;39(Suppl 1):S52-9. doi:10.2337/ dc16-S010

2 Christensen DH, Rungby J, Thomsen RW. Nationwide trends in glucose-lowering drug use, Denmark, 1999-2014. Clin Epidemiol 2016:8:381-7 doi:10.2147/CLEP.S113211

3 Azoulay L, Suissa S. Sulfonylureas and the Risks of Cardiovascular Events and Death: A Methodological Meta-Regression Analysis of the Observational Studies. Diabetes Care 2017;40:706-14. doi:10.2337/dc16-1943

4 Hemmingsen B, Schroll JB, Wetterslev J, et al. Sulfonylurea versus metformin monotherapy in patients with type 2 diabetes: a Cochrane systematic review and meta-analysis of randomized clinical trials and trial sequential analysis. CMAJ Open 2014;2:E162-75. doi:10.9778/ cmajo.20130073

5 Dluhy RG, McMahon GT. Intensive glycemic control in the ACCORD and ADVANCE trials. N Engl J Med 2008;358:2630-3. doi:10.1056/ NEJMe0804182

6 Optimal Use Reports CADTH. Second-and Third-Line Pharmacotherapy for Type 2 Diabetes - Update of CADTH 2010 Reviews - Project Protocol. Canadian Agency for Drugs and Technologies in Health, 2012.

7 Roumie CL, Greevy RA, Grijalva CG, et al. Association between intensification of metformin treatment with insulin vs sulfonylureas and cardiovascular events and all-cause mortality among patients with diabetes. JAMA 2014;311:2288-96. doi:10.1001/ jama.2014.4312

8 Roumie CL, Min JY, Greevy RA, et al. Risk of hypoglycemia following intensification of metformin treatment with insulin versus sulfonylurea. CMAI 2016;188:E104-12. doi:10.1503/ cmaj.150904

$9 \mathrm{Yu} \mathrm{OH}$, Yin H, Azoulay L. The combination of DPP-4 inhibitors versus sulfonylureas with metformin after failure of first-line treatment in the risk for major cardiovascular events and death. Can J Diabetes 2015;39:383-9. doi:10.1016/j. jcjd.2015.02.002

10 Sillars B, Davis WA, Hirsch IB, Davis TM. Sulphonylurea-metformin combination therapy, cardiovascular disease and all-cause mortality: the Fremantle Diabetes Study. Diabetes Obes Metab 2010;12:757. 65. doi:10.1111/j.1463-1326.2010.01230.x

11 Hippisley-Cox J, Coupland C. Diabetes treatments and risk of amputation, blindness, severe kidney failure, hyperglycaemia, and hypoglycaemia: open cohort study in primary care BMJ 2016;352:i1450. doi:10.1136/bmj.i1450

12 Evans JM, Ogston SA, Emslie-Smith A, Morris AD. Risk of mortality and adverse cardiovascular outcomes in type 2 diabetes: a comparison of patients treated with sulfonylureas and metformin. Diabetologia 2006;49:930-6. doi:10.1007/s00125-006-0176-9
13 UK Prospective Diabetes Study (UKPDS) Group. Effect of intensive blood-glucose control with metformin on complications in overweight patients with type 2 diabetes (UKPDS 34). Lancet 1998;352:854-65. doi:10.1016/S0140-6736(98)07037-8

14 UK Hypoglycaemia Study Group. Risk of hypoglycaemia in types 1 and 2 diabetes: effects of treatment modalities and their duration. Diabetologia 2007;50:1140-7. doi:10.1007/s00125-007-0599-y

15 Walley T, Mantgani A. The UK General Practice Research Database. Lancet 1997:350:1097-9. doi:10.1016/S0140-6736(97)04248-7

16 Herrett E, Thomas SL, Schoonen WM, Smeeth L, Hall AJ. Validation and validity of diagnoses in the General Practice Research Database: a systematic review. Br J Clin Pharmacol 2010;69:4-14. doi:10.1111/j.1365-2125.2009.03537.x

17 Douros A, Yin H, Yu OHY, Filion KB, Azoulay L, Suissa S. Pharmacologic Differences of Sulfonylureas and the Risk of Adverse Cardiovascular and Hypoglycemic Events. Diabetes Care 2017;40:1506-13. doi:10.2337/dc17-0595

18 Suissa S, Moodie EE, Dell'Aniello S. Prevalent new-user cohort designs for comparative drug effect studies by time-conditional propensity scores. Pharmacoepidemiol Drug Saf 2017;26:459-68. doi:10.1002/pds.4107

19 Schneeweiss S, Rassen JA, Glynn RJ, Avorn J, Mogun H, Brookhart MA. High-dimensional propensity score adjustment in studies of treatment effects using health care claims data. Epidemiology 2009;20:512-22. doi:10.1097/ EDE.0b013e3181a663cc

20 Herrett E, Shah AD, Boggon R, et al. Completeness and diagnostic validity of recording acute myocardial infarction events in primary care, hospital care, disease registry, and national mortality records: cohort study. BMJ 2013;346:f2350. doi:10.1136/bmj.f2350

21 Andrade SE, Harrold LR, Tjia J, et al. A systematic review of validated methods for identifying cerebrovascular accident or transient ischemic attack using administrative data. Pharmacoepidemiol Drug Saf 2012;21(Suppl 1):100-28. doi:10.1002/pds.2312

22 Abdelmoneim AS, Hasenbank SE, Seubert JM, Brocks DR, Light PE, Simpson SH. Variations in tissue selectivity amongst insulin secretagogues: a systematic review. Diabetes Obes Metab 2012;14:130-8. doi:10.1111/j.1463-1326.2011.01496.x

23 Gribble FM, Reimann F. Pharmacological modulation of K(ATP) channels. Biochem Soc Trans 2002;30:333-9. doi:10.1042/ bst0300333

24 Gribble FM, Reimann F. Sulphonylurea action revisited: the postcloning era. Diabetologia 2003;46:875-91. doi:10.1007/s00125003-1143-3

25 Krentz AJ, Bailey CJ. Oral antidiabetic agents: current role in type 2 diabetes mellitus. Drugs 2005;65:385-411. doi:10.2165/00003495-200565030-00005

26 Ding P, VanderWeele TJ. Sensitivity Analysis Without Assumptions. Epidemiology 2016;27:368-77. doi:10.1097/ EDE.0000000000000457

27 Lipsitch M, Tchetgen Tchetgen E, Cohen T. Negative controls: a tool for detecting confounding and bias in observational studies. Epidemiology 2010;21:383-8. doi:10.1097/ EDE.0b013e3181d61eeb

28 Solomon SD, Chew E, Duh EJ, et al. Diabetic Retinopathy,: A Position Statement by the American Diabetes Association. Diabetes Care 2017;40:412-8. doi:10.2337/dc16-2641

29 Frier BM. Hypoglycaemia in diabetes mellitus: epidemiology and clinical implications. Nat Rev Endocrinol 2014;10:711-22. doi:10.1038/nrendo.2014.170

30 Crowley MJ, Williams JWJr, Kosinski AS, D’Alessio DA, Buse JB. Metformin Use May Moderate the Effect of DPP-4 Inhibitors on Cardiovascular Outcomes. Diabetes Care 2017;40:1787-9. doi:10.2337/dc17-1528

31 Azoulay L, Schneider-Lindner V, Dell'aniello S, Schiffrin A, Suissa S. Combination therapy with sulfonylureas and metformin and the prevention of death in type 2 diabetes: a nested case-control study. Pharmacoepidemiol Drug Saf 2010;19:335-42. doi:10.1002/ pds. 1834

32 Summary of product characteristics-Glucophage 2017 updated 23.05.2017. https://www.medicines.org.uk/emc/medicine/20952

Appendix: Supplementary materials 